\title{
Imaging Synaptic Density: A Different Look at Neurologic Diseases
}

\author{
Eugenii A. Rabiner
}

Imanova Ltd. and Centre for Neuroimaging Sciences, Institute of Psychiatry, Psychology, and Neuroscience, King's College, London, United Kingdom

$\mathbf{L}$ rodegenerative pathology. The development of novel, diseasemodifying medication requires the ability to identify patients in early (even presymptomatic) stages of disease and follow the progress of the neurodegenerative process within individuals. Current methods used to evaluate synaptic loss in the human brain are limited to histology or electron microscopy of postmortem tissue. Such cross-sectional examinations have obvious drawbacks for drug development, are unsuitable for examining subjects in the early phases of the disease process, and lack the ability to follow the longitudinal pathology within individuals. Noninvasive molecular imaging techniques provide the obvious route for addressing these shortcomings. In the past, several candidate molecular targets were suggested for this role on the basis of the availability of suitable PET radioligands and the presence of the target molecules on glutamatergic or $\gamma$-aminobutyric acid-ergic neurons, which comprise large proportions of the total neuronal populations. These candidate targets include the $\gamma$-aminobutyric acid-A receptor $\left({ }^{11} \mathrm{C}\right.$-flumazenil), the 5-hydroxytryptamine receptors $1 \mathrm{~A}$ and 2A ( ${ }^{11} \mathrm{C}-W A Y-100635$ and $\left.{ }^{11} \mathrm{C}-\mathrm{MDL}-100907\right)$, and the histamine3 receptors $\left({ }^{11} \mathrm{C}-\mathrm{GSK}-189254\right.$ and $\left.{ }^{11} \mathrm{C}-\mathrm{MK}-8278\right)$.

The use of molecular imaging methods to quantify synaptic loss requires the identification of a molecular target with several suitable characteristics. To be useful as a general marker of synaptic density, such a target should be present on all synapses (rather than on just a subset). In addition, a stable stoichiometry should exist between the target and the synapse-a relationship that should remain consistent in the presence of a disease process. Finally, medications widely used in these patient populations should not have significant affinity for the molecular target in question. Hence, the recent development of PET ligands for the synaptic vesicle glycoprotein $2 \mathrm{~A}$ (SV2A), a target present in most (if not all) presynaptic terminals (1), represents an exciting breakthrough in our ability to monitor the synaptic status in the brain of preclinical species (2) and humans (3). SV2A belongs to the family of synaptic vesicle glycoproteins, which also include SV2B and SV2C, but SV2A is the only member of the family that is present ubiquitously in the adult brain, has a stoichiometry (with an estimated $\sim 1.5$ molecules per vesicle),

Received Sep. 4, 2017; revision accepted Oct. 30, 2017.

For correspondence or reprints contact: Eugenii A. Rabiner, Imanova Ltd., Burlington Danes Building, Hammersmith Hospital, Du Cane Rd., London, W12 ONN, U.K

E-mail: ilan.rabiner@imanova.co.uk

Published online Nov. 9, 2017.

COPYRIGHT @ 2018 by the Society of Nuclear Medicine and Molecular Imaging. DOI: 10.2967/jnumed.117.198317 and correlates well with classic markers of presynaptic terminals, such as synaptophysin and synaptotagmin. These characteristics make SV2A an excellent candidate marker of synaptic density.

Several SV2A-selective compounds have been labeled with PET isotopes and evaluated as radioligands for the SV2A. ${ }^{11} \mathrm{C}$ levetiracetam (4) demonstrated poor brain uptake and a low signal. ${ }^{11} \mathrm{C}$-UCB-A was tested in mini pigs, rats (5), and rhesus monkeys (Nabulsi, unpublished data, 2012) but showed slow brain kinetics limiting its utility as a PET imaging tool. Hence, most of the work in this area has been performed with ${ }^{11} \mathrm{C}-\mathrm{UCB}-$ $\mathrm{J}(2)$ and ${ }^{18} \mathrm{~F}-\mathrm{UCB}-\mathrm{H}(6)$, with both ligands demonstrating kinetics suitable for robust quantification of SV2A density. ${ }^{11} \mathrm{C}-\mathrm{UCB}-$ $\mathrm{J}$ has favorable dosimetry $(4.5 \mu \mathrm{Sv} / \mathrm{MBq})(2)$ and an excellent test-retest variability in the human brain $(3 \%-5 \%$ across the various regions) (7). ${ }^{18} \mathrm{~F}-\mathrm{UCB}-\mathrm{H}$ displays an adequate signal and a variability higher than ${ }^{11} \mathrm{C}$-UCB-J but similar to other radioligands in use (6), with estimated human radiation dosimetry of $19.7 \mu \mathrm{Sv} / \mathrm{MBq}(8)$.

A head-to-head comparison of ${ }^{11} \mathrm{C}-\mathrm{UCB}-\mathrm{J}$ and ${ }^{18} \mathrm{~F}-\mathrm{UCB}-\mathrm{H}$ in the nonhuman primate brain (2) demonstrated in vivo ${ }^{11} \mathrm{C}-\mathrm{UCB}-\mathrm{J}$ affinity approximately 9 -fold higher than that of ${ }^{18} \mathrm{~F}-\mathrm{UCB}-\mathrm{H}$ and a much higher specific signal. Because no reference region exists for $\mathrm{SV} 2 \mathrm{~A}$, the volume of distribution has to be used as an outcome parameter. For ${ }^{18} \mathrm{~F}-\mathrm{UCB}-\mathrm{H}$, approximately $50 \%$ of the volume of distribution comprises nondisplaceable binding, whereas the corresponding value for ${ }^{11} \mathrm{C}-\mathrm{UCB}-\mathrm{J}$ is closer to $20 \%$, providing a significant advantage for ${ }^{11} \mathrm{C}-\mathrm{UCB}-\mathrm{J}$ over ${ }^{18} \mathrm{~F}-\mathrm{UCB}-\mathrm{H}$. PET ligands labeled with ${ }^{11} \mathrm{C}$ have significant advantages over those labeled with ${ }^{18} \mathrm{~F}$, such as the ability to obtain more than 1 scan per day on the same subject (particularly valuable when radial artery cannulation is required or in studies involving pharmacologic or behavioral challenges) and significantly lower dosimetry, allowing multiple scanning of individuals. The superior imaging characteristics combined with an ${ }^{11} \mathrm{C}$ label make ${ }^{11} \mathrm{C}-\mathrm{UCB}-\mathrm{J}$ the current ligand of choice for investigating synaptic density in the brain, but ${ }^{18} \mathrm{~F}-\mathrm{UCB}-\mathrm{H}$ provides a viable option for smaller PET centers relying on radioligand distributed from central production sites.

To date, several pilot studies have examined SV2A density in a variety of clinical conditions. Hypothesized reductions in SV2A binding were seen around the epileptic focus in temporal lobe epilepsy patients (9) and in cortical areas of patients with mood disorders (10) and schizophrenia (11). ${ }^{11} \mathrm{C}$-UCB-J has also been used to characterize the relationship between the plasma concentration and SV2A occupancy of the antiepileptic drugs levetiracetam and brivaracetam (12). Of particular interest are pilot data indicating reductions in SV2A density consistent with synaptic loss in a patient with Alzheimer disease (Fig. 1) (13). These early results suggest that SV2A PET ligands have broad 


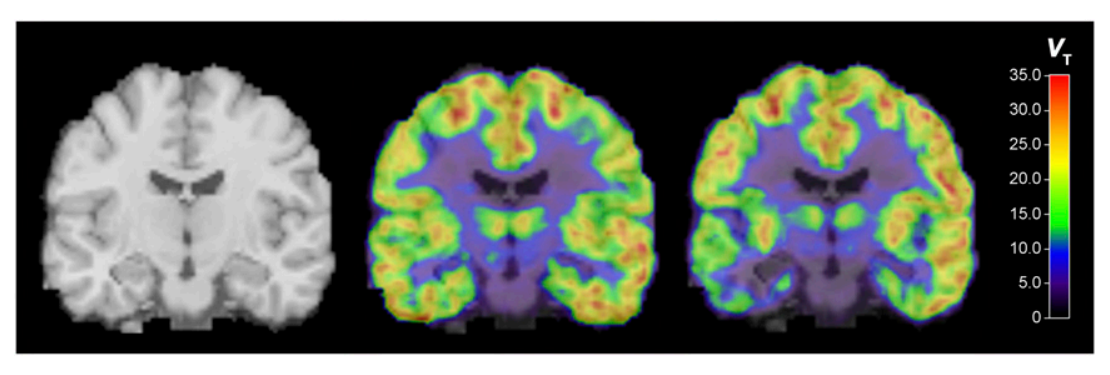

FIGURE 1. SV2A density (expressed as ${ }^{11} \mathrm{C}-\mathrm{UCB}-\mathrm{J}$ volume of distribution $\left[V_{\mathrm{T}}\right]$ ) in brain of patient with $A D$ (middle) and age-matched healthy control (right). Template MR image is provided to aid in anatomic localization (left). (Courtesy of Richard Carson, Yale University PET Center.)

\section{DISCLOSURE}

No potential conflict of interest relevant to this article was reported.

\section{REFERENCES}

1. Bajjalieh SM, Frantz GD, Weimann JM, McConnell SK, Scheller RH. Differential expression of synaptic vesicle protein 2 (SV2) isoforms. $J$ Neurosci. 1994;14:5223-5235.

2. Nabulsi NB, Mercier J, Holden D, et al. Synthesis and preclinical evaluation of ${ }^{11} \mathrm{C}$ UCB $\mathrm{J}$ as a PET tracer for imaging the synaptic vesicle glycoprotein 2A in the brain. $J$ Nucl Med. 2016;57:777-784.

3. Finnema SJ, Nabulsi NB, Eid T, et al. Imaging synaptic density in the living human brain. Sci Transl Med. 2016;8:348ra96.

applicability in the study of brain pathology, and several large PET programs are under way using SV2A as a molecular marker of neurodegenerative pathology in the human brain (e.g., MIND MAPS: https://mitochondrialdiseasenews.com/ 2017/04/05/imanova-mrc-funding-mind-maps-study/).

The future utility of SV2A radioligands as markers of synaptic density will depend largely on the demonstration of signal change correlating with disease progression. A better understanding of the underlying biology of the SV2A will be essential for the appropriate interpretation of changes seen on PET studies. Although the SV2A has a stoichiometry consistent with individual synaptic vesicles, information on the variability of vesicle numbers per individual presynaptic terminal - and the effects of pathology on the number of SV2As per vesicle, the number of vesicles per synapse, and the effective affinity of the PET ligands for SV2A-is needed. In addition, we need to understand whether acute changes in neuronal activity lead to a change in ${ }^{11} \mathrm{C}-\mathrm{UCB}-\mathrm{J}$ or ${ }^{18} \mathrm{~F}-\mathrm{UCB}-\mathrm{H}$ PET signal.

Methodologic improvements will enable access to these methods for a wider community of clinical PET researchers. Although the lack of a reference region necessitates the use of an arterial-blood input function for quantification of SV2A PET ligands, the potential exists for implementation of pseudoreference region approaches that may obviate the collection of arterial blood for some specific study designs (though more work is required to achieve this) (14). Although the utility of such methods remains to be demonstrated, they may be usefully applied in specific experimental situations in which relative regional, rather than global, absolute binding values are of interest, or when useful assumptions may be made about the levels of nondisplaceable binding (15). Alternative ${ }^{18} \mathrm{~F}$-labeled ligands are in development $(16,17)$ and may provide higher-signal options to ${ }^{18} \mathrm{~F}-\mathrm{UCB}-\mathrm{H}$ for groups restricted to ${ }^{18} \mathrm{~F}$-labeled compounds.

In conclusion, the development of good-quality PET ligands for the SV2A creates an exciting field of molecular imaging that has great prospects for the investigation and monitoring of brain pathology involving synaptic dysfunction.
4. Cai H, Mangner TJ, Muzik O, Wang MW, Chugani DC, Chugani HT. Radiosynthesis of ${ }^{11} \mathrm{C}$-levetiracetam: a potential marker for PET imaging of SV2A expression. ACS Med Chem Lett. 2014;5:1152-1155.

5. Estrada S, Lubberink M, Thibblin A, et al. $\left[{ }^{11} \mathrm{C}\right] \mathrm{UCB}-\mathrm{A}$, a novel PET tracer for synaptic vesicle protein 2A. Nucl Med Biol. 2016;43:325-332.

6. Warnock GI, Aerts J, Bahri MA, et al. Evaluation of ${ }^{18} \mathrm{~F}-\mathrm{UCB}-\mathrm{H}$ as a novel PET tracer for synaptic vesicle protein 2A in the brain. J Nucl Med. 2014;55:13361341 .

7. Finnema SJ, Nabulsi NB, Mercier J, et al. Kinetic evaluation of ${ }^{11} \mathrm{C}$ UCB-J, a novel radioligand for PET imaging of synaptic vesicle glycoprotein $2 \mathrm{~A}$ in humans. J Cereb Blood Flow Metab. January 1, 2017 [Epub ahead of print].

8. Bretin F, Bahri MA, Bernard C, et al. Biodistribution and radiation dosimetry for the novel SV2A radiotracer $\left[{ }^{18} \mathrm{~F}\right] \mathrm{UCB}-\mathrm{H}$ : first-in-human study. Mol Imaging Biol. 2015; 17:557-564.

9. Finnema S, Detyniecki K, Chen M-K, et al. Reduced SV2A binding in the seizure onset zone in temporal lobe epilepsy patients: a PET study with ${ }^{11} \mathrm{C}$ UCB-J. J Nucl Med. 2017;58:632-638.

10. Esterlis I, Pietrzak R, DellaGioia N, et al. In vivo evidence of lower synaptic density in depression and associated mood and cognitive deficits: a $\left[{ }^{11} \mathrm{C}\right] \mathrm{UCB}-\mathrm{J}$ PET imaging study [abstract]. Biol Psychiatry. 2017;81(suppl):S159. synaptic density in schizophrenia: a $\left[{ }^{11} \mathrm{C}\right]$ UCB-J PET imaging study [abstract]. Biol Psychiatry. 2017;81(suppl):S389.

12. Naganawa M, Finnema S, Mercier J, et al. Drug characteristics derived from PET kinetic modeling studies: combined ${ }^{11} \mathrm{C}-\mathrm{UCB}-\mathrm{J}$ human PET with levetiracetam and brivaracetam occupancy of SV2A [abstract]. J Nucl Med. 2017;58 (suppl):358.

13. Bao W, Jia H, Finnema S, Cai Z, Carson RE, Huang Y. PET imaging for early detection of Alzheimer's disease. from pathologic to physiologic biomarkers. PET Clin. 2017;12:329-350.

14. Rossano S, Finnema S, Fontaine K, Carson R. Optimizing OSEM reconstruction parameters for imaging synaptic density in humans with ${ }^{11} \mathrm{C}-\mathrm{UCB}-\mathrm{J}$ PET [abstract]. J Nucl Med. 2017;58(suppl):576.

15. Gunn RN, Murthy V, Catafau A, et al. Translational characterization of $\left[{ }^{11} \mathrm{C}\right]$ GSK931145, a PET Ligand for the Glycine Transporter Type 1. Synapse. 2011;65:1319-1332.

16. Li S, Cai Z, Zhang W, et al. Synthesis and in vivo evaluation of ${ }^{18} \mathrm{~F}-\mathrm{UCB}-\mathrm{J}$ : radiotracer for PET imaging of synaptic density [abstract]. J Nucl Med. 2017;58 (suppl):851.

17. Cai Z, Li S, Finnema S, et al. Imaging synaptic density with novel ${ }^{18}$ F-labeled radioligands for synaptic vesicle protein-2A (SV2A): synthesis and evaluation in nonhuman primates [abstract]. J Nucl Med. 2017;58(suppl):547.
11. Radhakrishnan R, Skosnik P, Finnema S, et al. In-vivo evidence of decreased 